\title{
INFLUENCE OF INCUBATION TEMPERATURE ON THE CAMP CONTENT IN HEART MUSCLE CELLS FROM CHICKEN EMBRYOS (GALLUS GALLUS F. DOMESTICUS)
}

\author{
Brauch, N, Tönhardt, H, Promny, T, v.Plettenberg, D \\ Institute of Veterinary-Physiology, Free University Berlin, Germany
}

cAMP is the second messenger for the $\beta_{1}$-adrenoreceptors. It effects the cellular response. The cAMP content in heart cells changes during embryonic development. In the past, Promny (2000) has made investigations with turkey embryos under short-term modifications of incubation temperature $\left(\mathrm{T}_{\mathrm{a}}\right)$ in our institute. The cAMP content of embryonic heart muscle cells on D25 is lower than on D23 in the three $\mathrm{T}_{\mathrm{a}}$-groups $\left(35,5^{\circ} \mathrm{C}, 37,5^{\circ} \mathrm{C}, 38,5^{\circ} \mathrm{C}\right)$. At $35,5^{\circ} \mathrm{C}$ there is a higher cAMP content on D25 compared with the other two $\mathrm{T}_{\mathrm{a}}$-groups on $\mathrm{D} 25$.

Here we examined the influence of long-term modifications of incubation temperature on basal cAMP content and its response to the sympathomimetic drug, Isoprenaline in developing white Legghorn eggs.

We considered the first day of incubation as D1. The incubation temperature was increased $\left(38,5^{\circ} \mathrm{C}\right)$ in the warm group and decreased $\left(35,0^{\circ} \mathrm{C}\right)$ in the cold group on D15. The control group was continously incubated at $37,5^{\circ} \mathrm{C}$. On D18 and D20, eggs of all groups were taken and the hearts were prepared. Heart tissue were digested with Trypsin to form the cell suspension. cAMP content was determined in the cells by means of cAMP enzyme immunoassay from BIOTRAK.

The basal cAMP content of embryonic heart muscle cells on D18 and D20 was similar in all three Ta-groups.
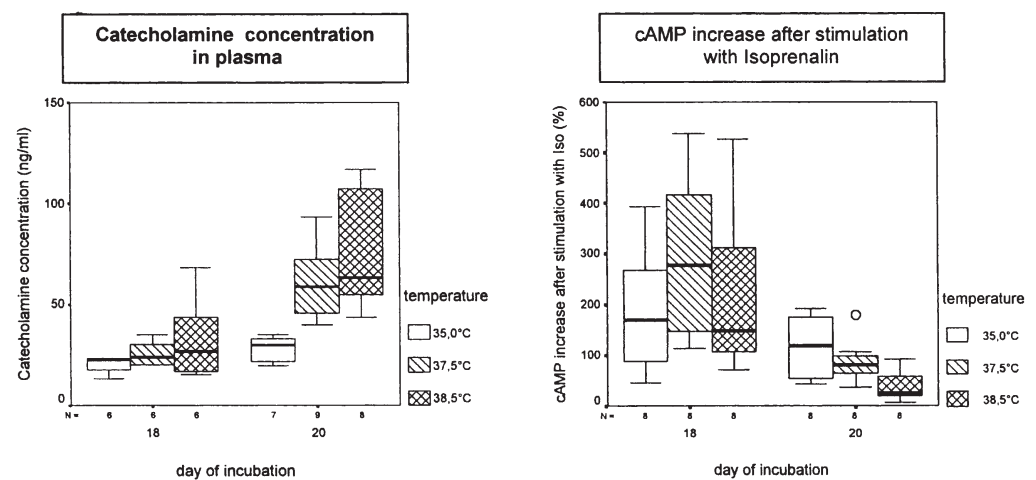

Intracellular cAMP content increased more in D18 embryos after stimulation with Isoprenaline than in D20 embryos. This reflects an age-dependent development of the regulation of cAMP content. Temperature-dependent differences in response to Isoprenaline tend to show larger increases in cAMP in the cold group and the control group compared with the changes in the warm group. These results along with results from earlier investigations on Catecholamine concentrations in chicken embryos (v.Plettenberg, 1999) show, that catecholamine levels rise and the cAMP-response to isoprenaline in the heart decreases from D18 to D20. The extent of the change in both, catecholamine and cAMP-response are similar in the three Ta-groups.

This study is supported by the H.W.Schaumann Foundation 